Research Article

\title{
Systems-Pharmacology-Based Identification of Antitumor Necrosis Factor Effect in Mimeng Flower Decoction for the Treatment of Diabetic Retinopathy
}

\author{
Yingzi Li, Ying Huang, and Changsen Tu $(D)$ \\ Eye Hospital, Wenzhou Medical University, WenZhou 325000, China \\ Correspondence should be addressed to Changsen Tu; tuchangsen2@163.com
}

Received 2 July 2019; Revised 14 September 2019; Accepted 22 October 2019; Published 19 November 2019

Academic Editor: Sakthivel Muniyan

Copyright (c) 2019 Yingzi Li et al. This is an open access article distributed under the Creative Commons Attribution License, which permits unrestricted use, distribution, and reproduction in any medium, provided the original work is properly cited.

\begin{abstract}
The traditional Chinese medicine of Mimeng flower decoction (MFD) is effective in treating diabetic retinopathy (DR), but the mechanism is still unclear. This study aims at investigating the mechanism of MFD in treating DR. First, active compounds in MFD were filtered out by the systems pharmacology method and used as bait to fish potential targets. The common genes between the targets and DR-related genes were selected to construct the compound-target-disease network and identify the network hub gene as a key gene. Molecular docking was simulated to assess the binding affinity of active compounds towards the gene protein. Streptozotocin- (STZ-) induced diabetic rat model was administered to evaluate the efficacy of MFD in treating DR and its effects on retinal gene expression. Finally, 53 active compounds were screened out from the seven herbs in MFD, with a total of 136 targets. After intersecting with 210 DR-related genes, 21 common genes were applied to construct the network, and tumor necrosis factor (TNF) was identified as the hub gene. The active compounds of acacetin, kaempferol, luteolin, and quercetin showed a good binding affinity towards TNF (C-score $\geq 4)$. In diabetic rats, MFD treatment reversed the retinal impairment and decreased retinal TNF expression significantly. In conclusion, this study adopted the method of systems pharmacology to screen out active compounds and construct the compound-target-disease network and found that MFD could ameliorate DR by downregulating the network hub gene of TNF.
\end{abstract}

\section{Introduction}

Diabetic retinopathy (DR) is one of the most common and serious complications in diabetes mellitus (DM), characterized by chronic and progressive retinal microvascular lesions [1]. It has become the leading cause of vision loss and blindness among working adults in developed countries [2]. Almost all patients of type $1 \mathrm{DM}$ (T1DM) and $60 \%$ of T2DM patients will develop visual impairment after a disease duration of 15 20 years [3]. DR greatly lowers the life quality of diabetic patients and causes a heavy burden to the society.

Traditional Chinese medicine (TCM) has been widely used in the treatment of DR, which was built on the medical practices of ancient Chinese scholars for more than 2500 years. According to the syndrome differentiation of TCM, DR is characterized by blood stasis and the deficiency of "qi" and "yin" [4]. TCM formulas usually comprise multiple herbs, which contain multiple active compounds and mediate various biological processes. Thus, TCM formulas exacted an outstanding role in the treatment of complicated diseases. Mimeng flower decoction (MFD) composes of seven herbs: Mimenghua (Latin name: Buddlejae Flos), Huanglian (Coptidis Rhizoma), Rougui (Cinnanmomi Cortex), Huangqi (Hedysarum Multijugum Maxim.), Nvzhenzi (Fructus Ligustri Lucidi), Wumei (Mume Fructus), and Yimucao (Leonuri Herba). According to the TCM theory, the decoction is characterized with invigorating blood circulation and tonifying "qi" and "yin," and thus, it was thought to be effective in treating DR. Although the efficacy has been implicated in several clinical trials, the bioactive ingredients and potential targets are still unclear [5-10].

In this study, we took a novel systems-pharmacologybased method to identify the molecular mechanism of MFD in treating DR. First, we filtered out the potential active 
components using the pharmacokinetic models. Then, the component-target and disease-target network was integrated, and network hub genes were experimentally validated (Figure 1).

\section{Methods}

2.1. Meta-Analysis of the Efficacy of MFD in Treating DR. Clinical trials focusing on the efficacy of MFD in treating DR were retrieved from the databases of PubMed, China Knowledge Resource Integrated Database (CNKI), and China Wanfang Database, using the keywords "Mimenghua" or "Miming flower" or "Buddlejae." Relative risks (RR) with 95\% confidence intervals (CI) were used to report the risk estimates following the Mantel-Haenszel method and random-effects model. The meta-analysis was performed by Review Manager 5.3.

\subsection{Screening Active Components in MFD and the Targets.} Traditional Chinese Medicines for Systems Pharmacology Database (TCMSP, https://5th.tcmspw.com/tcmsp.php) were searched for chemical ingredients of the seven herbs in MFD. Then, we screened the active constituents according to the ADME (absorption, distribution, metabolism, and excretion) principle, namely, drug-likeness (DL) $\geq 0.18$ and oral bioavailability $(\mathrm{OB}) \geq 30 \%$. Subsequently, these active components were used as bait to fish for the targets in TCMSP, which integrated the databases of DrugBank and HIT and the SysDT model [11]. The gene names were converted into official gene symbols using an R-script linking the website of PubMed (https://www.ncbi.nlm.nih. gov/gene/), and we only included human genes.

2.3. Screening DR-Related Genes. DR-related genes were obtained from Therapeutic Target Database (TTD, https:// db.idrblab.org/ttd/), DrugBank Database (https://www. drugbank.ca/), Pharmacogenomics Knowledgebase (PharmGKB, https://www.pharmgkb.org/), Comparative Toxicogenomics Database (CTD, https://www.pharmgkb. org/), and DisGeNET (https://www.disgenet.org/search). The gene identifiers were also converted into official gene symbols using an R-script linking the website of PubMed (https://www.ncbi.nlm.nih.gov/gene/), and we only included human genes.

2.4. Constructing Drug-Target-Disease Network and Identifying Hub Genes. The common genes between the targets of active compounds and DR-related genes were extracted to construct the drug-target-disease network, and the interactions between genes were based on the STRING database (https://string-db.org/). The parameters of degree, betweenness, and closeness in topological analysis were calculated to assess the centrality of each gene node in the network, and the hub genes were chosen as key genes in the treatment of MFD for DR. The network was visualized by Cytoscape 3.4.0, and the analysis was conducted by its plugin of NetworkAnalyzer.
2.5. Molecular Docking. Molecular docking analysis was conducted to evaluate the binding affinity of the active compounds towards the protein of hub gene. Crystal structure of tumor necrosis factor (TNF, PBD ID: 2AZ5) in complex with the small molecular inhibitor of $\mathrm{C}_{32} \mathrm{H}_{32} \mathrm{~F}_{3} \mathrm{~N}_{3} \mathrm{O}_{2}$ (PDD ID: 307) was retrieved from the Protein Data Bank (PDB) Database (https://www.rcsb.org/). To optimize the protein structure, we extracted the ligand, removed water, charged termini, and added hydrogens by using SYBYL-X 2.0 (Certara Inc., USA). Then, after generating the protomol in the prepared protein, the compounds were docked, respectively, into the binding site present in the protomol by employing the Surflex-Dock module in the SYBYL software. The consensus score (C-score) was used to assess the binding affinity, and the compounds with high C-score of 4 or 5 were classified as good docked [12].

2.6. Diabetic Rat Model. Fifty Sprague-Dawley (SD) rats of $8 \sim 10$ weeks and 200 250 grams were acquired from Beijing Weitong Lihua Experimental Animal Technology (Beijing, China). After one-week acclimatization, the rats were fed with a high-fat diet (HFD) for two weeks (Beijing Keao Xieli Feed, China) and then intraperitoneally injected with streptozotocin (STZ, $55 \mathrm{mg} / \mathrm{kg}$ ) (Sigma, USA) to induce T2DM. Some rats were served as normal control by feeding with the standard diet and injecting with physiological saline. The level of fasting blood glucose (FBG) was measured 3 days after the injection, and the rats with $\mathrm{FBG} \geq 16.7 \mathrm{mmol} / \mathrm{L}$ were considered as diabetic rats. The blood samples were collected from the tail vein. All experimental procedures and protocols were approved by the Ethics Committee of Eye Hospital of Wenzhou Medical University (2018016).

2.7. Treatment Protocols. The rats with $\mathrm{FBG} \geq 16.7 \mathrm{mmol} / \mathrm{L}$ were randomly divided into three groups: (1) treated with physiological saline by intragastric gavage (DR group); (2) treated with low-dose MFD $(5.9 \mathrm{~g} / \mathrm{kg}$.d, equivalent to 5 times the dose in humans, twice/day for 3 months) by intragastric gavage (low-dose MFD group); (3) treated with high-dose MFD (11.7 g/ kg.d, equivalent to 10 times the dose in humans, twice/day for 3 months) by intragastric gavage (high-dose MFD group). The herbs in MFD were purchased from Fujian Medicinal Materials (Fuzhou, China). After boiling in water for $30 \mathrm{~min}$, the decoction was filtered and collected.

2.8. Biochemical Measurements. The rats were anesthetized after the treatment, and blood samples were collected from the abdominal aorta. After $3000 \mathrm{rpm}$ centrifugation for $10 \mathrm{~min}$ at $4^{\circ} \mathrm{C}$, the serum was collected to measure FBG, aspartate aminotransferase (AST), and glutamic-pyruvic transaminase (ALT) using the automatic biochemical analyzer of AU480 (Beckman, USA) according to the kit instructions.

2.9. Retinal Histological Assessment. Retinas were fixed in $4 \%$ paraformaldehyde solution after isolation. Then, retinas were sectioned $(5 \mu \mathrm{m})$, stained with hematoxylin and eosin (H\&E), and observed under the microscope (Olympus, Japan). 


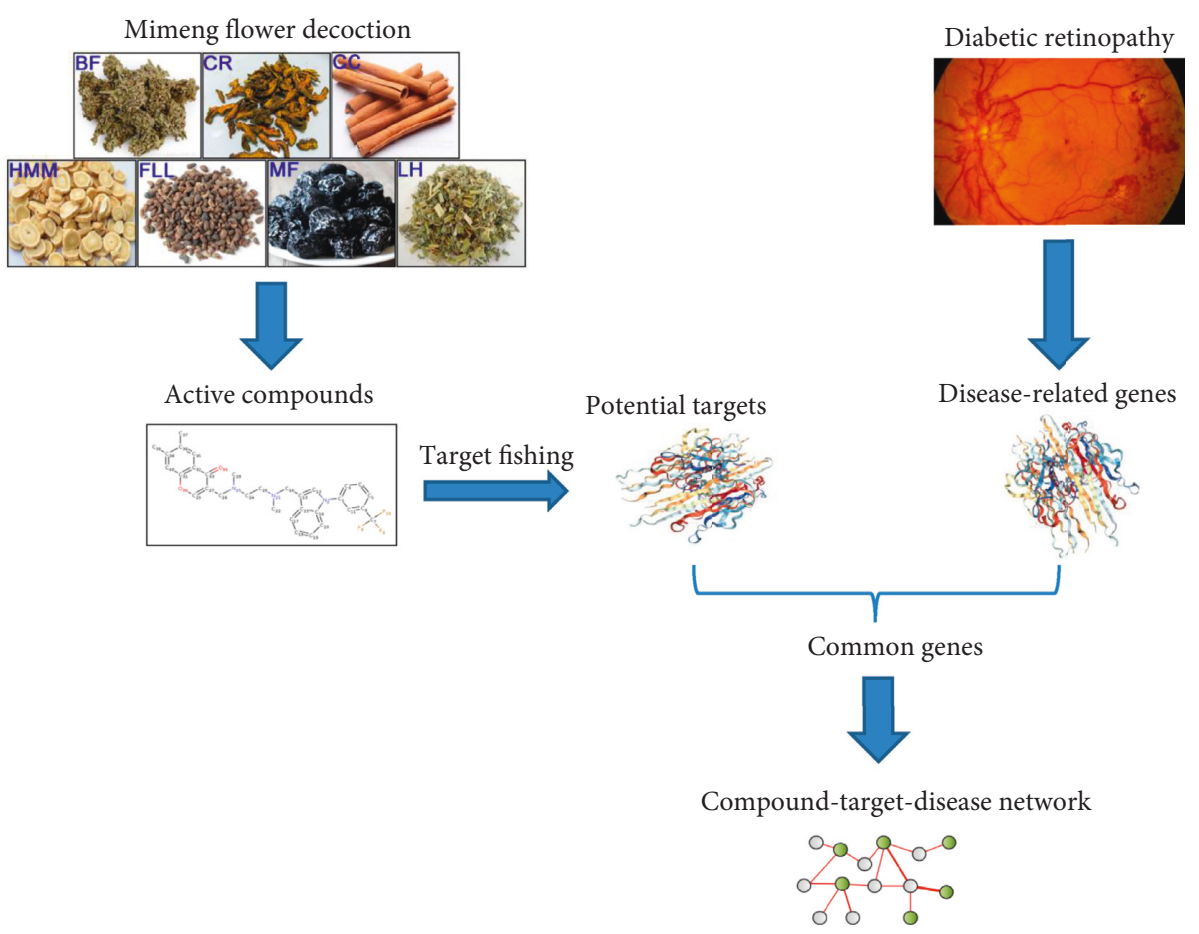

FIGURE 1: Flowchart of the systems pharmacology method in this study. BF, Buddlejae Flos; CR: Coptidis Rhizoma; CC, Cinnanmomi Cortex; HMM, Hedysarum Multijugum Maxim.; FLL, Fructus Ligustri Lucidi; MF, Mume Fructus; LH, Leonuri Herba.

2.10. Western Blot Analysis. Nuclear and cytosolic proteins in retinas were isolated as described in the extraction kits (BestBio Science, China). The protein concentration in each sample was normalized to the equal protein concentration. The protein samples were separated by SDS-PAGE (Sigma, USA) and then electrophoretically transferred onto the PVDF membrane (Millipore, USA). The membranes were incubated with primary and secondary antibodies (Abcam, UK). Immunoblots were visualized by the enhanced chemiluminescent kits (Sigma, USA). The grey densities of the protein bands were normalized by using $\beta$-actin or GAPDH density as an internal control, respectively.

\section{Results}

3.1. Identification of Active Compounds and the Targets and DR-Related Genes. In the pooled analysis, six clinical trials were reported an obvious efficacy of MFD in treating DR with a total of 449 MFD-treated patients and 460 controls, and the overall estimate of relative risk (RR) was $2.84(95 \%$ confidence interval (CI): 1.39 5.81) (Figure 2(a)) [5-10].

Then, ADME parameters were used to identify the potential active compounds in MFD, which consisted of seven herbs: Buddlejae Flos (BF), Coptidis Rhizoma (CR), Cinnanmomi Cortex (CC), Hedysarum Multijugum Maxim. (HMM), Fructus Ligustri Lucidi (FLL), Mume Fructus (MF), and Leonuri Herba (LH). According to the criteria of $\mathrm{DL} \geq 0.18$ and $\mathrm{OB} \geq 30 \%$, a total of 57 active compounds were identified (BF (4), CR (14), CC (0), HMM (20), FLL (13), MF (8), and LH (8)) (Figure 2(b); Table 1; Table S1).

Subsequently, these active components were used as bait to fish for the targets in TCMSP. After normalizing the gene names and removing duplication, we finally screened out a total of 136 targets (Figure 2(d); Table S2). The DR-related genes were searched in the databases of DrugBank, TTD, PharmGKB, CTD, and DisGeNET. After normalizing the gene symbols and removing duplication, we finally identified 210 DR-related genes.

3.2. Construction of Component-Target-Disease Network and Hub Gene Identification. Twenty-one common genes between the targets of active compounds and DR-related genes were selected to construct the compound-target-disease network by employing the protein-protein interaction (PPI) (Figures 2(c) and 2(e)). These genes were involved in multiple KEGG pathways, including AGE-RAGE signaling pathway in diabetic complications and TNF signaling pathway (Figure 3). In topological analysis of the network, tumor necrosis factor (TNF) had a significantly higher degree, closeness, and betweenness centrality than other gene nodes (Table 2). Thus, TNF was identified as the hub gene.

3.3. Binding Affinity of the Active Compounds towards TNF. In the previous analysis, TNF was targeted by four active compounds in MFD (acacetin, kaempferol, luteolin, and quercetin). To investigate whether these compounds could interact with TNF directly or not, molecular docking simulation of the interaction was conducted, respectively (Figure 4). Acacetin could form one hydrogen bond with the residue A/TYR151 of the TNF protein and showed a good binding affinity towards TNF $(\mathrm{C}$-score $=5)$. Kaempferol could form two hydrogen bonds with the residues A/ TYR151 and B/TYR151 and showed a good binding affinity 
TABle 1: Active compounds in Mimeng flower decoction.

\begin{tabular}{|c|c|c|c|}
\hline Mol ID & Molecular weight & Oral bioavailability (\%) & Drug-likeness \\
\hline MOL000006 & 286.25 & 36.16 & 0.25 \\
\hline MOL000033 & 428.82 & 36.23 & 0.78 \\
\hline MOL000098 & 302.25 & 46.43 & 0.28 \\
\hline MOL000211 & 456.78 & 55.38 & 0.78 \\
\hline MOL000239 & 314.31 & 50.83 & 0.29 \\
\hline MOL000296 & 414.79 & 36.91 & 0.75 \\
\hline MOL000354 & 316.28 & 49.60 & 0.31 \\
\hline MOL000358 & 414.79 & 36.91 & 0.75 \\
\hline MOL000371 & 314.36 & 53.74 & 0.48 \\
\hline MOL000374 & 642.67 & 41.72 & 0.69 \\
\hline MOL000378 & 316.38 & 74.69 & 0.30 \\
\hline MOL000379 & 462.49 & 36.74 & 0.92 \\
\hline MOL000380 & 300.33 & 64.26 & 0.42 \\
\hline MOL000387 & 418.38 & 31.10 & 0.67 \\
\hline MOL000392 & 268.28 & 69.67 & 0.21 \\
\hline MOL000398 & 316.33 & 109.99 & 0.30 \\
\hline MOL000417 & 284.28 & 47.75 & 0.24 \\
\hline MOL000422 & 286.25 & 41.88 & 0.24 \\
\hline MOL000433 & 441.45 & 68.96 & 0.71 \\
\hline MOL000438 & 302.35 & 67.67 & 0.26 \\
\hline MOL000439 & 626.67 & 49.28 & 0.62 \\
\hline MOL000442 & 314.31 & 39.05 & 0.48 \\
\hline MOL000449 & 412.77 & 43.83 & 0.76 \\
\hline MOL000622 & 266.37 & 63.71 & 0.19 \\
\hline MOL000762 & 510.52 & 35.36 & 0.65 \\
\hline MOL000785 & 352.44 & 64.60 & 0.65 \\
\hline MOL000953 & 386.73 & 37.87 & 0.68 \\
\hline MOL001040 & 272.27 & 42.36 & 0.21 \\
\hline MOL001418 & 376.54 & 61.02 & 0.38 \\
\hline MOL001420 & 412.77 & 38.00 & 0.76 \\
\hline MOL001421 & 334.50 & 85.97 & 0.33 \\
\hline MOL001422 & 334.50 & 66.29 & 0.33 \\
\hline MOL001439 & 304.52 & 45.57 & 0.20 \\
\hline MOL001454 & 336.39 & 36.86 & 0.78 \\
\hline MOL001458 & 320.34 & 30.67 & 0.86 \\
\hline MOL001689 & 284.28 & 34.97 & 0.24 \\
\hline MOL001790 & 592.60 & 39.84 & 0.71 \\
\hline MOL002668 & 334.37 & 45.83 & 0.87 \\
\hline MOL002894 & 322.36 & 35.74 & 0.73 \\
\hline MOL002897 & 336.39 & 43.09 & 0.78 \\
\hline MOL002903 & 339.42 & 55.37 & 0.77 \\
\hline MOL002904 & 351.38 & 36.68 & 0.82 \\
\hline MOL002907 & 404.55 & 104.95 & 0.78 \\
\hline MOL004576 & 304.27 & 57.84 & 0.27 \\
\hline MOL005043 & 400.76 & 37.58 & 0.71 \\
\hline MOL005146 & 568.63 & 48.87 & 0.71 \\
\hline MOL005147 & 406.47 & 54.41 & 0.47 \\
\hline MOL005169 & 486.86 & 40.23 & 0.82 \\
\hline MOL005190 & 288.27 & 71.79 & 0.24 \\
\hline MOL005195 & 450.48 & 83.12 & 0.80 \\
\hline MOL005209 & 401.60 & 30.11 & 0.75 \\
\hline MOL005211 & 696.87 & 65.45 & 0.23 \\
\hline MOL005212 & 404.55 & 103.23 & 0.78 \\
\hline MOL006673 & 468.84 & 46.04 & 0.83 \\
\hline MOL008601 & 318.55 & 46.90 & 0.23 \\
\hline MOL008647 & 313.38 & 86.71 & 0.26 \\
\hline MOL013352 & 454.56 & 43.29 & 0.77 \\
\hline
\end{tabular}

$(\mathrm{C}$-score $=4)$. Luteolin could form four hydrogen bonds with the residues A/TYR151, B/TYR151, A/GLY121, and A/ GLY122 and showed a good binding affinity (C-score $=4)$.
Quercetin could form two hydrogen bonds with the residues A/TYR151 and B/TYR151 and showed a good binding affinity $(\mathrm{C}$-score $=4)$. 


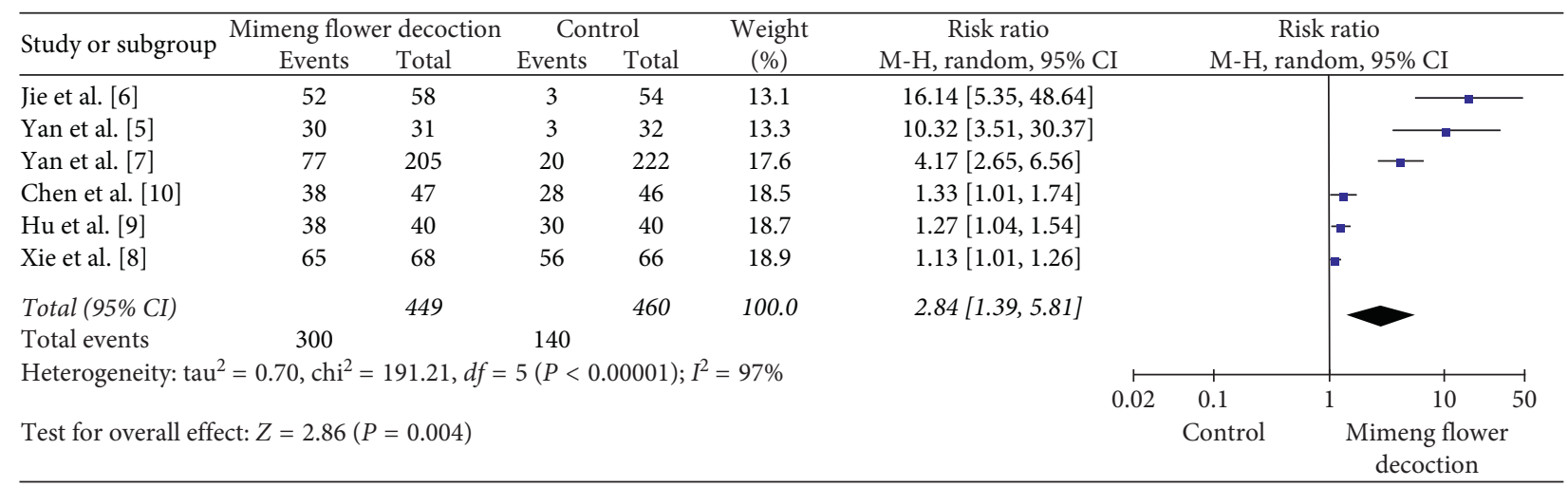

(a)

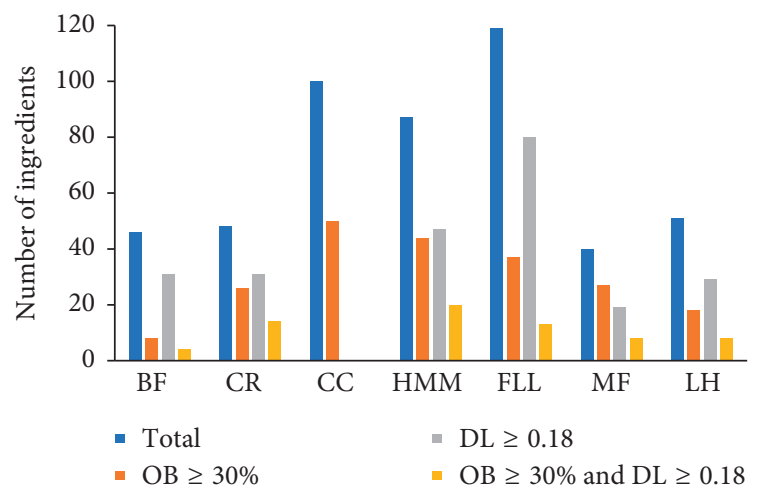

(b)

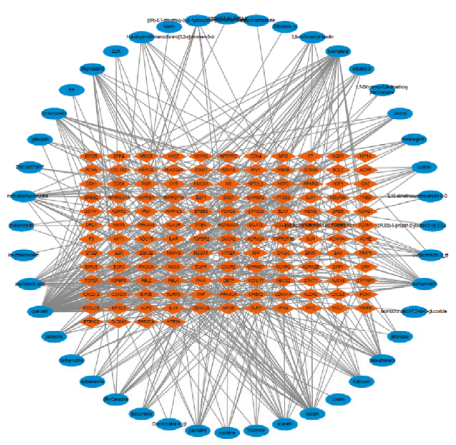

(d)
Targets of active compounds

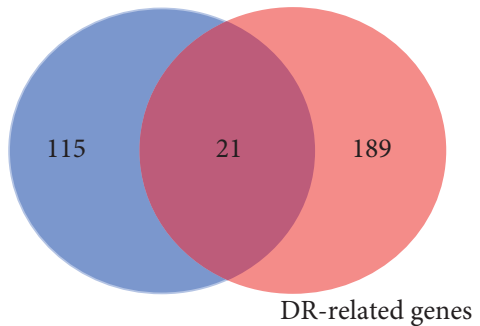

(c)

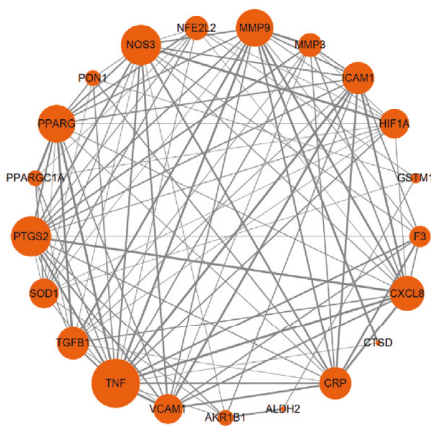

(e)

FIGURE 2: Systems pharmacology analysis of Mimeng flower decoction (MFD) in treating diabetic retinopathy (DR). (a) Meta-analysis of the efficacy of MFD in treating DR. (b) Screening active compounds in MFD. (c) Network of active compounds and the targets. Blue nodes represent active compounds, and orange nodes represent potential targets. The edge represents the interaction between them. (d) Overlap analysis of the targets of active compounds and DR-related genes. (e) Compound-target-disease network. The nodes represent the genes, and node size is proportional to its degree. The edges represent the interaction between genes, and edge size is proportional to the interaction score based on the STRING database. DL, drug-likeness; OB, oral bioavailability.

3.4. Efficacy of MFD in Treating Diabetic Rats. STZ-induced diabetic rats $(n=10)$ had a significant decrease in body weight $(P<0.001)$ and a significant increase in FBG, ALT, and AST $(P<0.001)$ when compared with the healthy controls (HCs) $(n=10)$ (Figure $5(\mathrm{a}))$. After the administration of MFD for 3 months, the rats in both low-dose and high-dose MFD groups had a significant increase in body weight $(P<0.05)$ and a significant decrease in FBG, ALT, and AST $(P<0.001)$ when compared with the diabetic rats ( $n=10$ per group). No significant difference was detected between the low-dose and high-dose MFD groups $(P>0.05)$.
These results indicated the hypoglycemic and hepatoprotective effects of MFD in treating diabetes.

In retinal histological assessment, the diabetic rats had a larger number of vessels in the ganglion cell layer (GCL), inner nuclear layer (INL), and outer nuclear layer (OPL), and a thinner retina, GCL, and nerve fiber layer (NFL) than the HCs (Figure 5(b)). After MFD treatment, the vessel number decreased and the retinal thickness increased, but no significant difference was detected between the low-dose and high-dose MFD groups. This indicated the efficacy of MFD in treating DR. 
TABLE 2: Topological analysis of the compound-target-disease network.

\begin{tabular}{|c|c|c|c|c|}
\hline Gene symbol & Gene name & Degree & Closeness & Betweenness \\
\hline TNF & Tumor necrosis factor & 18 & 0.91 & 0.15 \\
\hline NOS3 & Nitric oxide synthase 3 & 15 & 0.80 & 0.05 \\
\hline PTGS2 & Prostaglandin-endoperoxide synthase 2 & 15 & 0.80 & 0.05 \\
\hline MMP9 & Matrix metallopeptidase 9 & 14 & 0.74 & 0.05 \\
\hline PPARG & Peroxisome proliferator-activated receptor-gamma & 14 & 0.74 & 0.03 \\
\hline CXCL8 & C-X-C motif chemokine ligand 8 & 13 & 0.71 & 0.02 \\
\hline ICAM1 & Intercellular adhesion molecule 1 & 12 & 0.69 & 0.02 \\
\hline CRP & C-reactive protein & 12 & 0.69 & 0.02 \\
\hline TGFB1 & Transforming growth factor beta 1 & 12 & 0.67 & 0.01 \\
\hline SOD1 & Superoxide dismutase 1 & 11 & 0.69 & 0.06 \\
\hline HIF1A & Hypoxia-inducible factor 1 subunit alpha & 11 & 0.67 & 0.01 \\
\hline VCAM1 & Vascular cell adhesion molecule 1 & 11 & 0.65 & 0.00 \\
\hline NFE2L2 & Nuclear factor, erythroid 2 like 2 & 9 & 0.65 & 0.04 \\
\hline MMP3 & Matrix metallopeptidase 3 & 9 & 0.61 & 0.00 \\
\hline F3 & Coagulation factor III, tissue factor & 8 & 0.59 & 0.00 \\
\hline AKR1B1 & Aldo-keto reductase family 1 member B & 6 & 0.59 & 0.07 \\
\hline PON1 & Paraoxonase 1 & 6 & 0.59 & 0.02 \\
\hline PPARGC1A & PPARG coactivator 1 alpha & 6 & 0.57 & 0.00 \\
\hline GSTM1 & Glutathione S-transferase mu 1 & 4 & 0.48 & 0.02 \\
\hline $\mathrm{ALDH} 2$ & Aldehyde dehydrogenase 2 family member & 2 & 0.41 & 0.00 \\
\hline CTSD & Cathepsin D & 2 & 0.50 & 0.00 \\
\hline
\end{tabular}

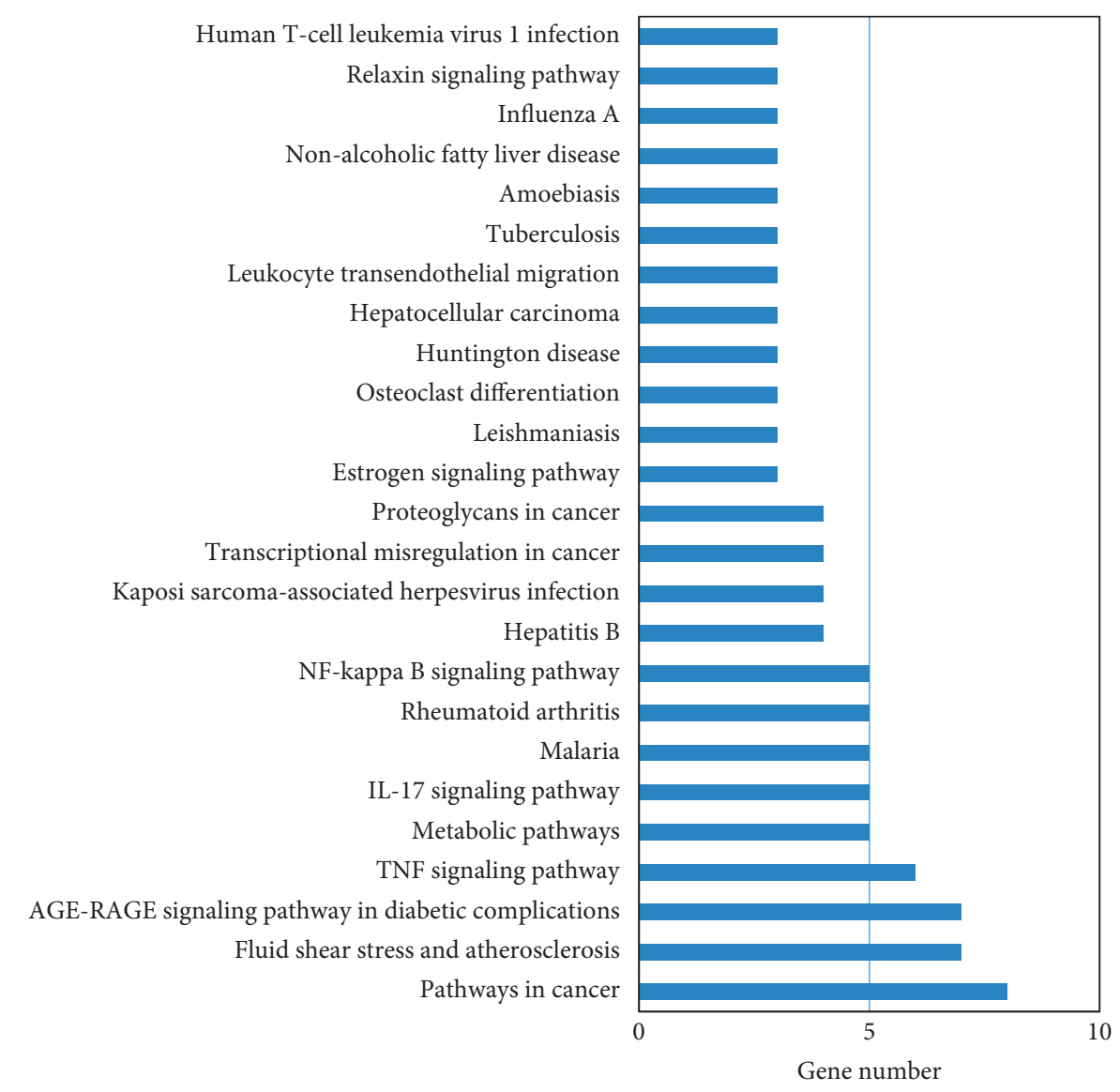

FIGURE 3: KEGG pathway analysis of the genes in the compound-target-disease network.

3.5. Effects of MFD Treatment on Retinal TNF Expression in Diabetic Rats. The diabetic rats had a higher retinal expression of TNF than the HCs $(P<0.05)$ (Figure 5(c)). After
MFD treatment, TNF expression decreased significantly $(P<0.05)$, but no obvious difference was detected between the low-dose and high-dose MFD groups $(P>0.05)$. 


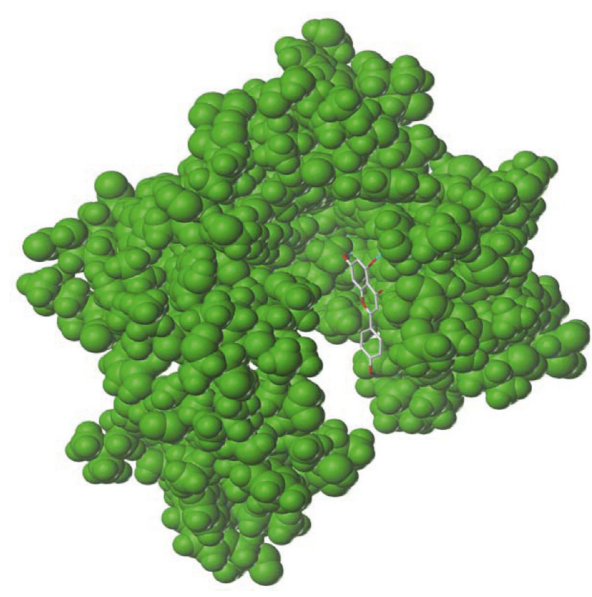

(a)

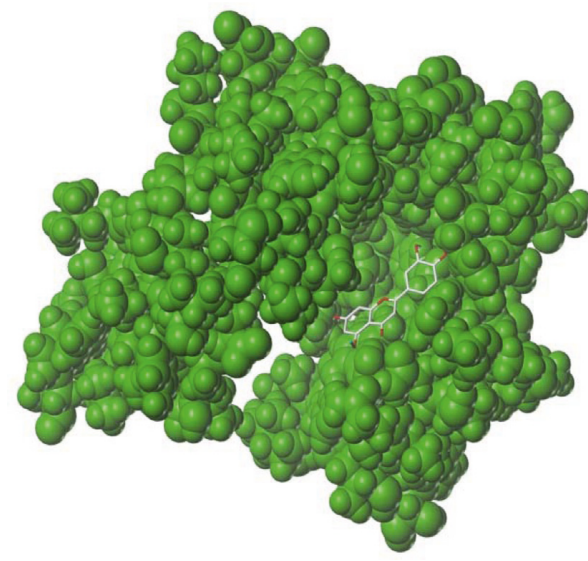

(c)

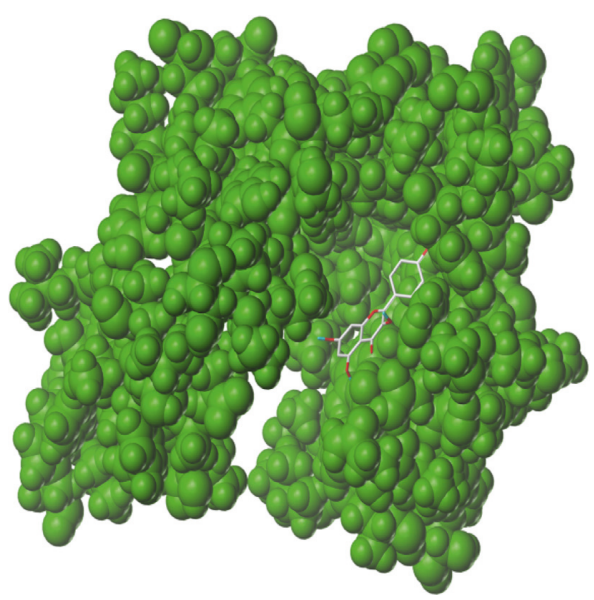

(b)

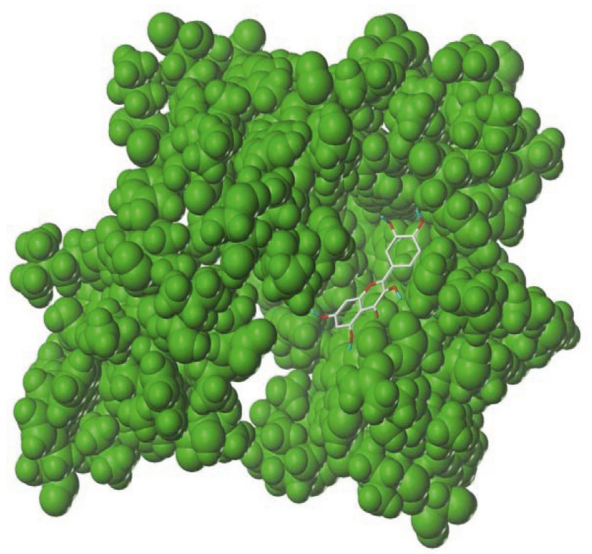

(d)

Figure 4: Molecular docking analysis of the binding affinity of active compounds (acacetin, kaempferol, luteolin, and quercetin) towards the target of tumor necrosis factor (TNF). C-score, consensus score. (a) Acacetin C-score =4; (b) kaempferol C-score =4; (c) luteolin Cscore $=4$; $(\mathrm{d})$ quercetin $\mathrm{C}$-score $=4$.

\section{Discussion}

DR is one of the most serious complications in DM, and it is characterized by blood stasis and the deficiency of "qi" and "yin" according to the theory of TCM. MFD has a good performance in invigorating blood circulation and tonifying "qi" and "yin," and thus, it was thought to be effective in treating DR. In our meta-analysis of clinical trials, it showed a good efficacy in DR treatment. However, the molecular mechanism is still unclear. As a complex of several herbs, we do not know which ingredient works and the potential targets.

To explore the mechanism, we adopted the methods of systems pharmacology. First, active compounds were filtered out and used as bait to fish potential targets. The DR-related genes were obtained by the systematic search. Finally, 21 common genes were identified to construct the compoundtarget-disease network. Among the 21 genes, multiple genes have been reported in the pathogenesis of DR. For example, the genotypes of NOS3 rs869109213 polymorphism were associated with the risk of DR [13]. High glucose-induced hyperosmolarity promoted PTGS2 (COX-2) expression and angiogenesis [14]. MMP9 was implicated in retinal capillary cell apoptosis and subsequent development of DR [15]. PPARG has been strongly indicated as a primary target in the treatment of DR [16].

In the KEGG pathway analysis, these genes were associated with multiple pathways, such as AGE-RAGE signaling pathway in diabetic complications, TNF signaling pathway, IL-17 signaling pathway, and NF-kappa B signaling pathway, indicating the involvement of inflammatory signaling pathways in the development of DR. These genes might be potential therapeutic targets for DR. It has been found that IL-17A could exacerbate DR by impairing Müller cell function, and blocking IL-17A could alleviate DR $[17,18]$.

In topological analysis of the compound-target-disease network, TNF showed a significant network centrality than other gene nodes, and it was identified as the hub gene. TNF$\alpha$ has been demonstrated to be involved in a variety of intraocular inflammatory diseases via the proinflammatory effects $[19,20]$. Furthermore, the active compounds in MFD showed a good binding affinity towards TNF, indicating that MFD might exert the therapeutic role by targeting TNF. 

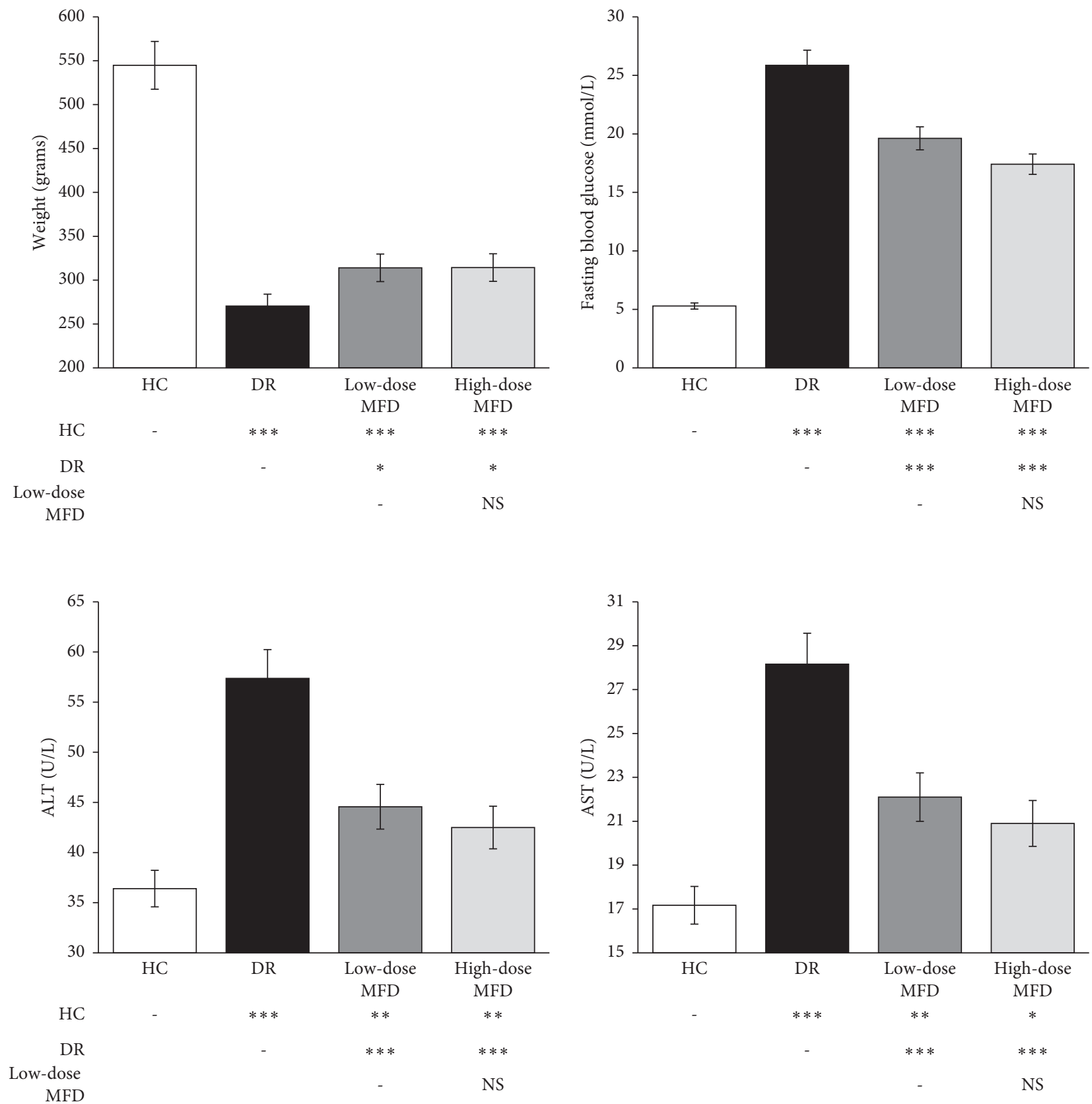

(a)

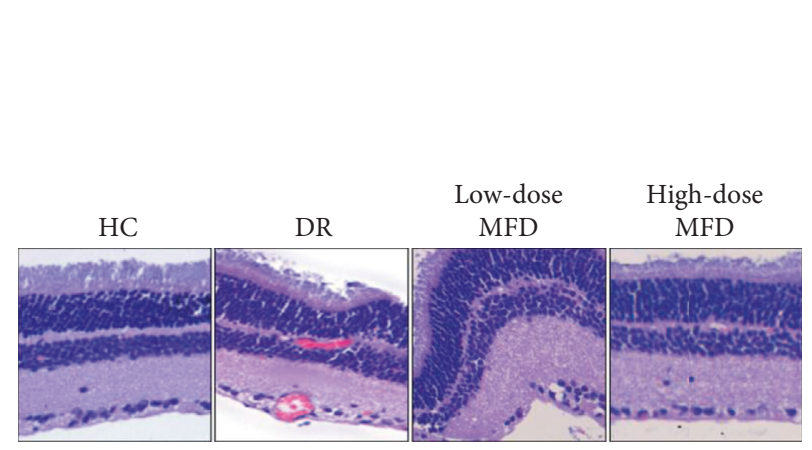

(b)

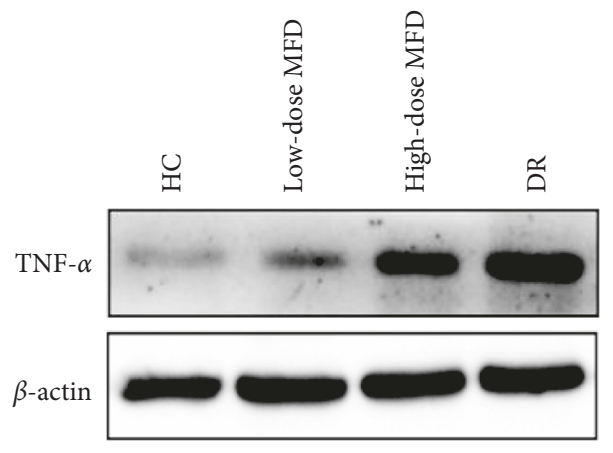

(c)

FiguRE 5: Efficacy of Mimeng flower decoction (MFD) in treating diabetic rats and its effect on the retinal expression of tumor necrosis factor (TNF). (a) Effects of MFD treatment on body weight, fasting blood glucose, ALT, and AST in diabetic rats $(n=10$ per group). (b) Retinal histological assessment of MFD treatment in diabetic rats. (c) Effects of MFD treatment on retinal TNF expression in diabetic rats. $\mathrm{DR}$, diabetic retinopathy; $\mathrm{HC}$, healthy control. ${ }^{*} \mathrm{P}<0.05,{ }^{* *} \mathrm{P}<0.01,{ }^{* * *} \mathrm{P}<0.01$. 
In an animal experiment, MFD showed a good efficacy in treating diabetic rats and reversing retinal impairment, which was consistent with the clinical trials. The TNF expression also decreased significantly after MFD treatment, but no dose-response relationship was found. This might contribute to the dose interval or treatment duration which was not large or long enough to magnify the difference. In the clinical study of Xia et al., fundus fluorescein angiography (FFA) found a significant decrease of new vessels among the patients supplemented with one-month or twomonth MFD treatment when compared with the control, but no obvious difference was detected between the one-month and two-month groups (effective rates: $89.7 \%$ vs. $95.6 \%$, $P>0.05$ ) [8]. Similarly, in the clinical study of Chen et al., optical coherence tomography (OCT) detected a significant decrease of macular edema in the MFD treatment groups, but no obvious difference was detected between the onemonth and two-month groups (314.57 \pm 109.51 vs. $308.64 \pm 130.57, P>0.05)$ [10]. Thus, we thought the effective dose of MFD was easily available, but it might need several times the effective dose to reach a better efficacy, which was not recommended considering the adverse effects (Figure 5).

\section{Conclusion}

This study adopted the method of systems pharmacology to screen out active compounds and construct the compoundtarget-disease network and found that MFD could ameliorate DR by downregulating the network hub gene of TNF.

\section{Data Availability}

The data used to support the findings of this study are available from the corresponding author upon request.

\section{Conflicts of Interest}

The authors declare no conflicts of interest.

\section{Acknowledgments}

This study was supported by a grant from Eye Hospital, Wenzhou Medical University.

\section{Supplementary Materials}

Table S1: Mimeng flower decoction comprising of seven herbs. Table S2: the active compounds in Mimeng flower decoction and their target genes. (Supplementary Materials)

\section{References}

[1] D. S. W. Ting, G. C. M. Cheung, and T. Y. Wong, "Diabetic retinopathy: global prevalence, major risk factors, screening practices and public health challenges: a review," Clinical \& Experimental Ophthalmology, vol. 44, no. 4, pp. 260-277, 2016.

[2] N. Cheung, P. Mitchell, and T. Y. Wong, "Diabetic retinopathy," The Lancet, vol. 376, no. 9735, pp. 124-136, 2010.
[3] D. S. Fong, L. P. Aiello, F. L. Ferris III, and R. Klein, "Diabetic retinopathy," Diabetes Care, vol. 27, no. 10, pp. 2540-2553, 2004.

[4] C. Ou, Y. J. Yang, and Q. H. Peng, "Yiqi Yangyin Huoxue method in treating diabetic retinopathy: a systematic review and meta-analysis," Evidence-based Complementary and Alternative Medicine, vol. 2019, Article ID 6020846, 7 pages, 2019.

[5] J. Yan, J. S. Gao, C. H. Jie, J. T. Song, Z. Z. Wu, and X. L. Guo, "Effects of Mimenghua formula on TCM symptoms of early diabetic retinopathy and safety," Journal of Beijing University of Traditional Chinese Medicine, vol. 33, no. 11, pp. 773-776, 2010.

[6] C. H. Jie, J. S. Gao, J. Yan et al., "Effect of Buddleia prescription on the visual function in non-proliferative diabetic retinopathy," Journal of Traditional Chinese Ophthalmology, vol. 20, no. 6, pp. 323-325, 2010.

[7] J. Yan, J. S. Gao, C. H. Jie et al., "Impacts on fundus lesions in non-proliferative diabetic retinopathy treated with Flos Buddlejae formula," World Journal of Integrated Traditional and Western Medicine, vol. 8, no. 3, pp. 246-248, 2013.

[8] W. Xia, Y. L. Chen, and X. Y. Kong, "Clinical observation of panretinal photocoagulation combined with buddleja officinalis formula on the treatment of severe non-proliferative diabetic retinopathy," Hebei Journal of Traditional Chinese Medicine, vol. 38, no. 7, pp. 992-994, 2016.

[9] X. D. Hu, F. Ren, and Y. Nan, "Clinical observation on treating non proliferative diabetic retinopathy with Mimeng flower prescription and calcium dobesilate capsules," Modern Journal of Integrated Traditional Chinese and Western Medicine, vol. 26, no. 18, pp. 1945-1947, 2017.

[10] Y. L. Chen, Y. Y. Kong, W. Xia, and L. Xie, "Clinical observation of 71 cases of severe nonproliferative and stage IV diabetic retinopathy treated with Mimeng flower decoction combined with $532 \mathrm{~nm}$ laser photocoagulation," Contemporary Medicine, vol. 23, no. 11, pp. 130-132, 2017.

[11] J. Ru, P. Li, J. Wang et al., "TCMSP: a database of systems pharmacology for drug discovery from herbal medicines," Journal of Cheminformatics, vol. 6, no. 1, p. 13, 2014.

[12] M. Thillainayagam, K. Malathi, A. Anbarasu, H. Singh, R. Bahadur, and S. Ramaiah, "Insights on inhibition of Plasmodium falciparum plasmepsin I by novel epoxyazadiradione derivatives-molecular docking and comparative molecular field analysis," Journal of Biomolecular Structure \& Dynamics, vol. 37, no. 12, pp. 3168-3182, 2019.

[13] D. Bregar, I. Cilenšek, S. Mankoč, A. Reschner, D. Petrovič, and M. Globočnik, "The joint effect of the endothelin receptor B gene (EDNRB) polymorphism rs10507875 and nitric oxide synthase 3 gene (NOS3) polymorphism rs869109213 in Slovenian patients with type 2 diabetes mellitus and diabetic retinopathy," Bosnian Journal of Basic Medical Sciences, vol. 18, no. 1, pp. 80-86, 2017.

[14] R. Madonna, G. Giovannelli, P. Confalone, F. V. Renna, Y. J. Geng, and R. De Caterina, "High glucose-induced hyperosmolarity contributes to COX-2 expression and angiogenesis: implications for diabetic retinopathy," Cardiovascular Diabetology, vol. 15, no. 1, p. 18, 2016.

[15] M. Mishra, J. Flaga, and R. A. Kowluru, "Molecular mechanism of transcriptional regulation of matrix metalloproteinase-9 in diabetic retinopathy," Journal of Cellular Physiology, vol. 231, no. 8, pp. 1709-1718, 2016.

[16] V. Costa and A. Ciccodicola, "Is PPARG the key gene in diabetic retinopathy?," British Journal of Pharmacology, vol. 165, no. 1, pp. 1-3, 2012. 
[17] A. W. Qiu, Z. Bian, P. A. Mao, and Q. H. Liu, "IL-17A exacerbates diabetic retinopathy by impairing Müller cell function via Act1 signaling," Experimental \& Molecular Medicine, vol. 48, no. 12, p. e280, 2016.

[18] A.-W. Qiu, Q.-H. Liu, and J.-L. Wang, "Blocking IL-17A alleviates diabetic retinopathy in rodents," Cellular Physiology and Biochemistry, vol. 41, no. 3, pp. 960-972, 2017.

[19] M. Croft and R. M. Siegel, "Beyond TNF: TNF superfamily cytokines as targets for the treatment of rheumatic diseases," Nature Reviews Rheumatology, vol. 13, no. 4, pp. 217-233, 2017.

[20] B. Q. Guo, J. B. Xu, M. Xiao, M. Ding, and L. J. Duan, "Puerarin reduces ischemia/reperfusion-induced myocardial injury in diabetic rats via upregulation of vascular endothelial growth factor A/angiotensin-1 and suppression of apoptosis," Molecular Medicine Reports, vol. 17, no. 5, pp. 7421-7427, 2018. 


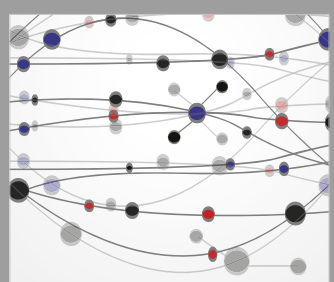

The Scientific World Journal
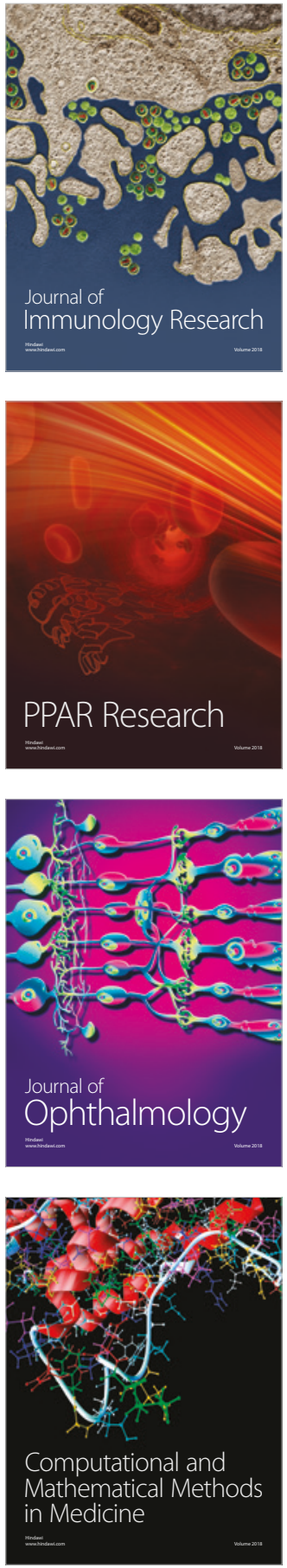

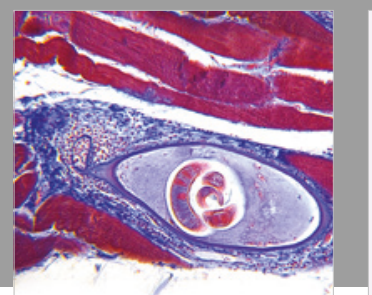

Gastroenterology Research and Practice

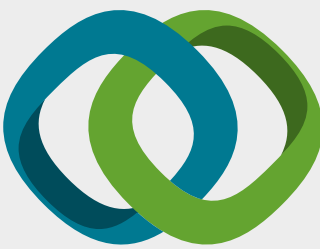

\section{Hindawi}

Submit your manuscripts at

www.hindawi.com
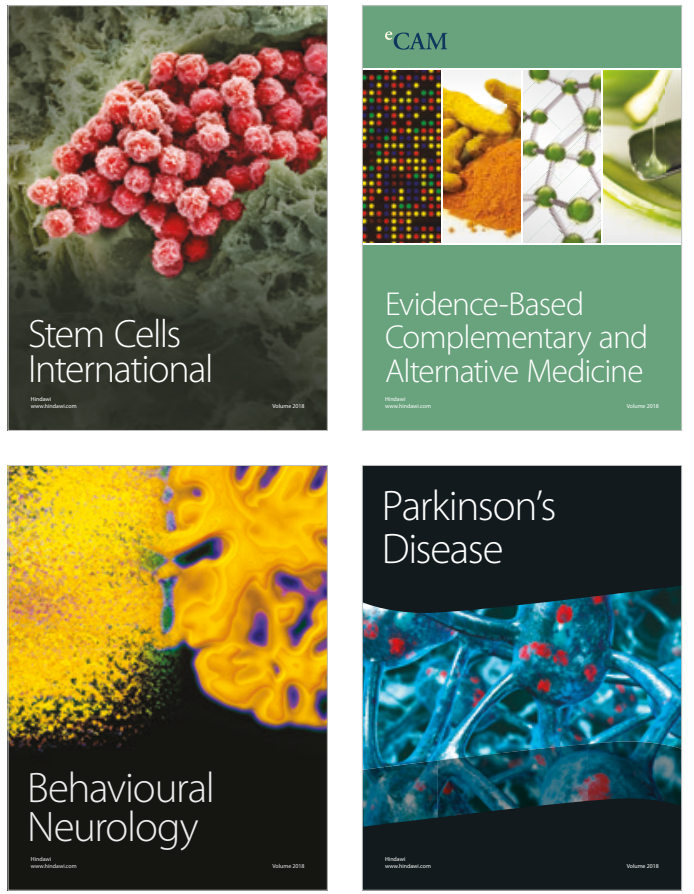

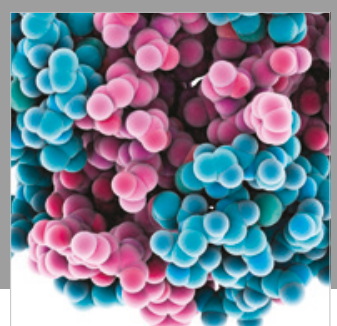

ournal of

Diabetes Research

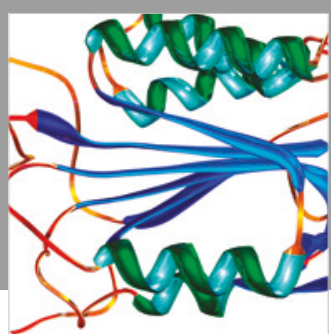

Disease Markers
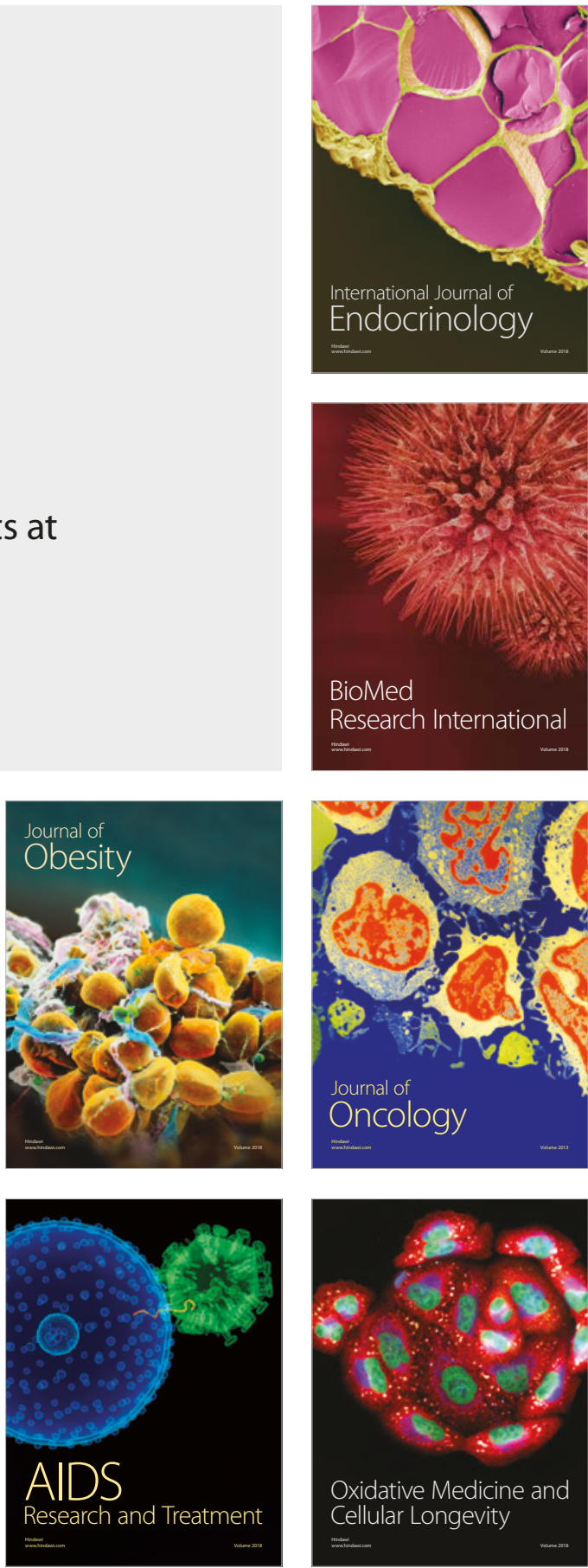